\title{
Substance Index for Volume 69
}

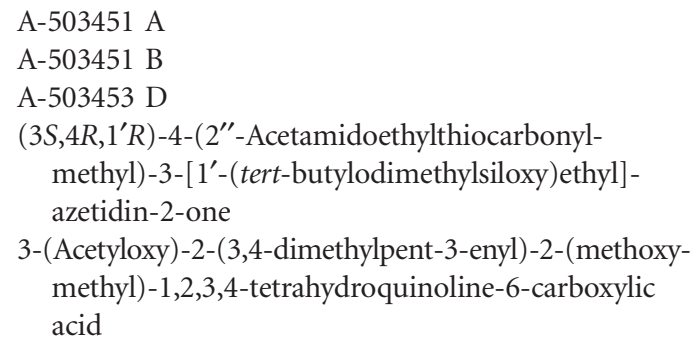

Benzyl (3-(Cbz)amino)propyl-(4-(Boc)aminobutyl)carbamate

Benzyl (4-aminobutyl)-(3-(Cbz)aminopropyl)carbamate

(2R,3S,4S)-4-Benzyloxy-2-(hydroxymethyl)-3-methyl3-vinylcyclohexan-1-one

(7R,8S)-8-Benzyloxy-7-methyl-1,4-dioxaspiro[4.5] decan-7-ol

(7S,8S)-8-(Benzyloxy)-7-methyl-1,4-dioxaspiro[4.5]decan-7-ol

(S)-4-Benzyloxy-3-methylcyclohex-2-en-1-one

$\left(2 R, 3 S, 5 R, 6 S, 1^{\prime} R\right)$-3-Benzyloxy-6-[1'-(tert-butyldimethylsiloxy)ethyl]-2-methoxycarbonyl-1-azabicyclo[3.2.0]heptan-7-one

(21S,14E,18E)-21-Benzyloxymethoxydocosa-14,18dien-1-ol

(21S,14E,18E)-21-Benzyloxymethoxydocosa-14,18dienal

$\left(2 R, 3 S, 5 R, 6 S, 1^{\prime} R\right)-6$ - [1'-(tert-Butyldimethylsiloxy)ethyl]-3-hydroxy-2-methoxycarbonyl-1-azabicyclo[3.2.0] heptan-7-one

$\left(2 R, 5 R, 6 S, 4^{\prime} R\right)-6$ - [1'-(tert-Butyldimethylosiloxy) ethyl]-2-methoxycarbonyl-1-azabicyclo[3.2.0] heptan-3,7-di-one

(2S,3S)-3-(Benzyloxy)-2-((Bnzyloxy)methyl)-3,4dihydro-2H-pyrrole-1-oxide

(2S,3S)-3-Benzyloxy-2-hydroxymethyl-3,4dihydro- $2 \mathrm{H}$-pyrrole- $\mathrm{N}$-oxide
747, 754 $\left(2 S, 3 S, 5 R, 6 R, 1^{\prime} R\right)$-3-Benzyloxy-6-[1' - (tert-butyldimethylosiloxy)ethyl]-2-(hydroxymethyl)-1-azabicyclo[3.2.0]heptan-7-one

(2S,3S,5R,6S, 1'R)-3-Benzyloxy-6-[1'-(tert-butyldimethylosiloxy)ethyl]-2-(hydroxymethyl)-1-azabicyclo[3.2.0]heptan-7-one

$(2 S, 4 E, 8 E)$-2-Benzyloxymethoxy-22-tert-butyldimethylsilyloxy-docosa-4,8-diene

$(3 R, 4 S)$-4-Benzyloxy-3-methyl-3-vinylcyclohexan-1-one Berberine

Berkleasmin $\mathrm{F}$

(S)-6-((Boc)amino)-2- [7-( $N, N^{\prime}$-Bis $(\mathrm{Cbz})$ guanidinoheptanamido $)]$ hexanoic acid

(S)-N-(6-(Boc)amino-1-(4-(1,3-Bis(Cbz)aminopropylamino)butylamino)-1-oxohexan-2-yl)-7-( $N, N^{\prime}-$ Bis $(\mathrm{Cbz})$ guanidino)heptanamide

tert-Butyl 4-(tetrahydropyrimidin-1(2H)-yl)butylcarbamate

Ceftazidime

Chelerythrine

Chloramphenicol

3-Chloro-5-(2-hydroxy-3-((2-methylpropan-2-ylamino)propoxy)-9H-xanthen-9-one

3-Chloro-5-(2-hydroxy-3-(1-hydroxy-3-methylbutan2-ylamino)propoxy)-9H-xanthen-9-one

3-Chloro-5-(2-hydroxy-3-(propan-2-ylamino)propoxy)-9H-xanthen-9-one

3-Chloro-5-(3-(1-hydroxy-2-methylpropan-2-ylamino)propoxy)-9H-xanthen-9-one

Chloromonilicin

Chlorotonil A

Cinanthrenol A, ent-(+)-

Ciprofloxacin

Citrinadin A

CL-55

Cladosporinone

Clindamycin

Coccoquinone A, B

Codonopsinine

Codonopsinine, 4-epi-(-)-

Curromycin A

Cy3 Apoptolidin A, H

Dalbavancin

Daptomycin

Dehydroevodiamine

6a,7-Didehydro-1,9,10-trihydroxy-2-methoxy-6methylaporphiniumtrifluoroacetate

6,6'-Dihydroxy-(1,1'-biphenyl)-3,3'-dicarboxylic acid 108

3,6-Dihydroxy-2-propylbenzaldehyde 111

2-(3,4-Dimethylpent-3-enyl)-2-(methoxymethyl)-3- 747 [(methylsulfonyl)oxy]-1,2,3,4-tetrahydroquinoline6-carboxylic acid

Drimentine I 
Ecumicin

EM900

Emefuran A, B $B_{2}, C_{1}, C_{2}, D$

Emefuranone A, farnesyl

Emefuranone $A_{1}, A_{2}, B$

Emefuranone B, farnesyl

Emefuranone C, farnesyl

Emericelloxide

Epoxyfilipin V, $16 \alpha, 17 \alpha-$

Epoxyfilipin V, $16 \beta, 17 \beta$ -

Eravacycline

Erythromycin A 11-N,12-O-cyclic carbamate derivatives, 11-[3[(arylcarbamoyl)oxy] propylamino]-11deoxy-6-O-methyl-3-oxo

(3S,16E,20E,23S)-Ethyl 23-benzyloxymethoxy-3hydroxytetracosa-16,20-dienoate

(3S,16E,20E,23S)-Ethyl 23-benzyloxymethoxy-3hydroxytetracosa-16,20-dienthioate

(3S,16E,20E,23S)-Ethyl 23-hydroxy-3-(p-methoxybenzyloxy)tetracosa-16,20-dienoate

Eushearilide

Eushearilide, (3S,16E,20E,23S)-(+)-

Euvesperin A, B

FD-891

FD-891, 10-deoxy-

FD-891, 25-O-demethyl-

FD-891, 25-O-methyl

4-(3-(4-(2-Fluorophenyl)piperazin-1-yl)-2hydroxypropoxy)-9H-xanthen-9-one dihydrochloride

Folipastatin, 7-chloro

Fumiquinazoline C, 2'-epi-

Fumiquinazoline D, 2'-epi-

Fusidic Acid

Gentamicin

Glycidylfilipin III, 15-

Haenamindole

Hangtaimycin

Hermitamide A, (-)-

Hermitamide B, (-)-

Hetiamacin B, C, D

(3S,16E,20E,23S)-23-Hydroxy-3-(p-methoxybenzyloxy)tetracosa-16,20-dienoic acid

(S)-7-Hydroxy-7-methyl-1,4-dioxaspiro[4.5]decan8-one

$(7 R, 8 S)$-7-Hydroxy-7-methyl-1,4-dioxaspiro[4.5]decan-8-yl acetate

(7S,8R)-7-Hydroxy-7-methyl-1,4-dioxaspiro[4.5]decan-8-yl dodecanoate

$(3 S, 16 E, 20 E, 23 S)-3-H y d r o x y-23$-methyltricosa-16,20dienolide

3-Hydroxy-2-oxo-2H-chromene-4,6-dicarboxylic acid

3-(2-Hydroxy-3-(prop-2-en-1-ylamino)propoxy)-5methyl-9H-xanthen-9-one

3-(2-Hydroxy-3-(propan-2-ylamino)propoxy)-5methyl-9H-xanthen-9-one

Iminimycin A

Intervenolin
Inthomycin B

Ircinal A

411

340

JBIR-76, -77

707

Kalkipyrone 348

Kendomycin 203

L-681,217, demethyl

Latifolian A 344

Leucinostation A, B 411

Levofloxacin 381

Lincomycin, 7(S)-7-(5-amino-1,3,4-thiadiazol-2- 368 ylthio)-7-deoxy

Lincomycin, 7(S)-7-(6-aminobenzo[d]thiazol-2- 368 ylthio)-7-deoxy

Lincomycin, 7(S)-7-(azetidine-3-ylthio)-7-deoxy 440

Lincomycin, 7(S)-7-deoxy-7-(4-((S)-2-dimethylami- 428 nomethylpyrrolidinocarbonyl)phenylthio)

Lincomycin, 7(S)-7-deoxy-7-(4-((S)-2-methoxymethylpyrrolidinocarbonyl)phenylthio)

Lincomycin, 7(S)-7-deoxy-7-[1-(piperidin-1-yl)azetidine-3-ylthio]

Lincomycin, 7(S)-7-deoxy-7-(4-morpholinocarbonylphenylthio)

Lincomycin, 7(S)-7-deoxy-7-(5-phenyl-1,3,4-thiadiazol-2-ylthio)

Lincomycin, 7(S)-7-deoxy-7-\{1-(morpholin-4-yl) carbonyl]azetidine-3-ylthio $\}$

Linezolid

Lumizinone A, B

Lyngbic acid, (-)-

Magnocurarine

Maniwamycin C, D, E, F

Manzamine A

MBJ-0110

4-(3-(2-Methoxybenzylamino)-2-hydroxypropoxy)9H-xanthen-9-one

(3S,16E,20E,23S)-3-(p-Methoxybenzyloxy)-23-methyltricosa-16,20-dienolide

4-(3-(3,4-Methoxyphenyl)ethylamino-2-hydroxypropoxy)-9H-xanthen-9-one hydrochloride

(7R,8S)-7-Methyl-1,4-dioxaspiro[4.5]decane-7,8-diol

(7RS,8SR)-7-Methyl-1,4-dioxaspiro[4.5]decane7,8-diol

(7S,8R)-7-Methyl-1,4-dioxaspiro[4.5]decane-7,8 -diol

(5R,6S, 1'R)-Methyl 3-[(2-acetamidoethyl)thio]-6[1'-(tert-butyldimethylsiloxy)ethyl)-7-oxo-1-azabicyclo[3.2.0] hept-2-ene-2-carboxylate

Methylated catechins

Milbemycin $\beta 3,13 \alpha$-hydroxy

Milbemycin $\beta 3,13 \alpha$-hydroxy-25-ethyl

Milbemycin $\beta 3,13 \alpha$-hydroxy-4-ethyl

Myrothecine C, dihydro

428

440

428

368

440

381

616

337

344

395

340

66

825

697

825

273

273

164

299

104

104

104

652

$N$-((S)-1-(4-(3-aminopropylamino)butylamino)-1oxo-6-(5-(2-oxo-hexahydro-1H-thieno[3,4- $d]$ imidazol-4-yl)pentanamido)hexan-2-yl)-7guanidinoheptanamide 
N-((S)-1-amino-10,17,24-trioxo-28-(2-oxo-hexahydro- $1 H$-thieno[3,4-d] imidazol-4-yl)-4,9,16,23-

tetraazaoctacosan-11-yl)-7-guanidinoheptanamide NBRI16716 A, B, C

NBRI23477 A, B

NFAT-133

Nitrofurantoin

NVB333

Oxysporizoline

Paraphaeosphaeride D

Penicyrone A, B

Pheganomycin

Phencomycin, 5,10-dihydro

Phorboxazole A, B

Phthoxazolin A

PM100117, 100118

Pyrrocidine A

Quinocitrinine A, B

Quinovosamycin

Ramizol

Resorcinomycin

Reveromycin A, A1a, A1b, A1c, A2a

Reveromycin A2a, C15-dehydro

Reveromycin A3a

Reveromycin $\mathrm{T}$

Reveromycin T ethylester

Rifampicin
183,791
Rocheicoside A $\quad 51$

RQN-18690A 121

Sagamilactam $\quad 818$

Sanguinarine $\quad 344$

Sansanmycin H, [4-BrPhe]- $\quad 765$

Sansanmycin H, [4-ClPhe]- $\quad 765$

Sansanmycin H, [4-MeOPhe]- $\quad 765$

Sansanmycin H, [4-MePhe]- $\quad 765$

Spirohexaline $\quad 798$

Streptanoate 124

Subglutinol C, D 561

Tabouensinium chloride $\quad 344$

Theaflavin 299

Triclosan $\quad 791$

1,9,10-Trihydroxy-2-methoxy-6-methylaporphinium 344 trifluoroacetate

Trimethoprim $\quad 791$

Tubuphenylalanine 294

Tubuvaline 294

Unantimycin A 456

$\begin{array}{ll}\text { Vancomycin } & 381,791\end{array}$

Verrulactone D, E $\quad 114$

$\begin{array}{ll}\text { Viridicatumtoxin } & 798\end{array}$

$\begin{array}{lr}\text { Xylarinol C } & 89\end{array}$

Yessotoxin $\quad 259$

Yoshinone A, B1, B2 348 